\title{
Percepciones de los estudiantes sobre sus vivencias en las prácticas clínicas
}

\section{Students' perceptions about their experiences in their clinical practices}

\section{Percepciones dos estudantes sobre suas vivências nas práticas clínicas}

\author{
E. García-Carpintero Blas ${ }^{\mathrm{a}{ }^{*}}$, J. Siles-González ${ }^{\mathrm{b} 2}$, M.E. Martínez-Roche ${ }^{\mathrm{c} 3}$, E. Martínez- \\ Miguel $^{\mathrm{d} 1}$, C. Manso-Perea ${ }^{\mathrm{e} 1}$, S. González-Cervantes ${ }^{\mathrm{f1}}$, E. García-García ${ }^{\mathrm{g} 1}$ \\ ORCID: \\ a $0000-0002-4984-2511 \quad{ }^{\mathrm{b}} 0000-0003-3046-639 \mathrm{X} \quad{ }^{\mathrm{c}} 0000-0001-8807-823 \mathrm{X} \quad{ }^{\mathrm{d}} 0000-0002-5153-5860$ \\ e0000-0001-5266-7868 ${ }^{\mathrm{f}} 0000-0002-9058-6126 \quad{ }^{\mathrm{g}} 00000-0002-9646-4840$
}

${ }^{1}$ Escuela Universitaria de Enfermería de la Cruz Roja, Universidad Autónoma de Madrid, Madrid, España ${ }^{2}$ Departamento de Enfermería, Facultad ciencias de la Salud, Universidad de Alicante, Alicante, España ${ }^{3}$ Departamento de Enfermería, Universidad de Murcia, Murcia, España

Recibido: 12 agosto 2018

Aceptado: 30 mayo 2019

\section{Resumen}

Introducción: Los estudiantes de enfermería deben adquirir conocimientos, habilidades, actitudes y valores, para proporcionar cuidados de calidad. Las prácticas clínicas son el contexto ideal para lograr algunas de las competencias requeridas.

Objetivo: Explorar las percepciones de los estudiantes sobre sus vivencias durante sus prácticas clínicas. Método: Abordaje metodológico descriptivo de tipo cualitativo y con un encuadre sociocrítico. Se analizaron los 45 portafolios de estudiantes de $4^{\circ}$ curso de la Escuela Universitaria de Enfermería (EUE) Cruz Roja, donde explican sus vivencias en prácticas. Se optó por un portafolio semiestructurado en función de la competencia a demostrar definiendo previamente las tareas o evidencias Se realizó un análisis de contenido que comprendía las siguientes fases: reducción de los datos; categorización-codificación, presentación de datos y conclusiones/verificación de los datos. Se alcanzó saturación en la información sobre las categorías presentadas. 
Resultados: Del análisis de los datos emergen distintas unidades de significado que se engloban en tres categorías: aprendizaje en prácticas, dificultades del entorno, crecimiento personal y profesional.

Conclusiones: El estudio evidencia la importancia de las prácticas clínicas para los estudiantes dentro del plan de estudios, ya que es donde aplican lo que han aprendido en el aula y son conscientes de la realidad a la que se enfrentan. El entorno clínico y la relación que se establezca entre este y el estudiante determina en muchas ocasiones el aprendizaje. Queda patente que los estudiantes durante sus prácticas clínicas toman conciencia de aquellos conocimientos del saber, saber hacer y saber estar.

Palabras clave: Estudiantes de enfermería; prácticas clínicas; investigación cualitativa; España.

\section{Abstract}

Introduction: Nursing students need specific knowledge, skills, attitudes and values in order to provide quality of care; and the clinical practices represent an ideal context to develop some of these required competences.

Objective: To explore the perceptions of nursing students about their own experiences in their clinical practices.

Method: This a qualitative descriptive study with social and critical focus. 45 practice-files from nursing students in the 4th course of a Mexico Red Cross School of Nursing were analyzed considering the following stages: data reduction, categorization-coding, data presentation, and data verification and conclusions. Data saturation was reached from the identified categories.

Results: From the data analysis, diverse units of meaning emerged within three main categories: learning in practices, difficulties in the environment, and personal and professional growth.

Conclusions: The study highlights the importance of clinical practices since it is there where students apply in real life what they learn in the classroom. The relationship between the student and the clinical environment greatly determines the quality of learning. So, it is suggested that students keep in mind reflecting on their to know, to know how, and to know how to be.

Keywords: Students, nursing; clinical practices; qualitative research; Spain.

\section{Resumo}

Introdução: Os estudantes de enfermagem devem adquirir conhecimentos, habilidades, atitudes e valores, para proporcionar cuidados de qualidade. As práticas clínicas são o contexto ideal para atingir algumas das competências requeridas.

Objetivo: Explorar as percepções dos estudantes sobre suas vivências durante suas práticas clínicas.

Método: Abordagem metodológica descritiva de tipo qualitativo e com um enfoque sociocrítico. Analisaram-se os 45 portfolios de estudantes de $4^{\circ}$ curso da Escuela Universitaria de Enfermería (EUE) Cruz Roja, onde explicam suas vivências em práticas. Optou-se por um portfólio semiestruturado em função da competência a demostrar definindo previamente as tarefas ou evidências. Realizou-se uma análise de conteúdo que compreendia as seguintes fases: redução dos dados; categorização-codificação, apresentação de dados e conclusões/verificação dos dados. Atingiu-se saturação na informação sobre as categorias apresentadas.

Resultados: Da análise dos dados emergem distintas unidades de significado que abrangem três categorias: aprendizagem em práticas, dificuldades do entorno, crescimento pessoal e profissional.

Conclusões: O estudo evidencia a importância das práticas clínicas para os estudantes dentro do plano de estudos, já que é onde aplicam o que aprenderam na aula e são conscientes da realidade à que se enfrentam. O entorno clínico e a relação que se estabeleça entre este e o estudante determina em muitas ocasiões a aprendizagem. É evidente que os estudantes durante suas práticas clínicas tomam consciência daqueles conhecimentos do saber, saber fazer e saber estar.

Palavras chave: Estudantes de enfermagem; práticas clínicas; pesquisa qualitativa; Espanha. 


\section{Introducción}

Los estudiantes de enfermería deben adquirir conocimientos, habilidades, actitudes y valores para proporcionar cuidados de calidad. Las prácticas clínicas son el contexto ideal para lograr algunas de las competencias requeridas ${ }^{1}$. Para ello, deben aplicar los conocimientos aprendidos en la enseñanza teórica a situaciones concretas del cuidado y desarrollar las actitudes necesarias para su futura profesión. Zabalza², afirma que las prácticas clínicas permiten aproximar a los estudiantes a escenarios profesionales reales, para generar marcos de referencia o esquemas cognitivos, de forma tal que los aprendizajes académicos queden iluminados por su sentido y su naturaleza en la práctica profesional.

Las prácticas en el Programa para obtener el Grado en Enfermería constituyen un campo de aprendizaje en el cuál convergen objetivos educativos de varias asignaturas. Estos objetivos están integrados, de tal forma, que el estudiante debe ser capaz de aplicar los diferentes conocimientos, habilidades y actitudes en los sucesivos contextos clínicos en los que desarrolle su proceso de enseñanza-aprendizaje, desde una perspectiva holística, superadora de toda fragmentación o compartimentación estanca ${ }^{3}$. La práctica clínica sigue siendo un recurso muy valioso en la preparación de los estudiantes, de apoyo a la integración de la teoría y la práctica, y que une el «saber qué» con el «saber cómo».

Las prácticas clínicas, aunque ocasionan gran satisfacción para los estudiantes, al mismo tiempo, son una fuente de estrés para ellos. En este nuevo escenario de aprendizaje se enfrentan a un sinfín de situaciones, personas, enfermedades, etc., provocándoles lo que muchos autores denominan como shock de la realidad, que vendría provocado por la disonancia entre cómo se cree que deberían ser las cosas y como son en realidad ${ }^{4-6}$. Para reducir esta situación es importante que la esencia del aprendizaje se base en la incorporación que hace el estudiante a sus experiencias y estructuras mentales de las nuevas realidades del contexto. Ya que es él quien debe utilizar los medios a su alcance para integrar la teoría y la práctica, de una manera dinámica y reducir la diversidad encontrada.

Los profesionales docentes debemos investigar sobre aspectos relacionados con el proceso de enseñanza-aprendizaje, tanto de las metodologías docentes y evaluadoras como de la experiencia de aprendizaje de nuestros estudiantes, durante las prácticas clínicas. Estudios previos han abordado este análisis desde la perspectiva de los métodos de evaluación y aprendizaje en prácticas ${ }^{1,4,7}$, sobre los factores estresantes durante las mismas ${ }^{8,9}$, el proceso de tutorización ${ }^{10}$ y el ambiente de aprendizaje ${ }^{11-13}$. También, existen estudios relacionados con la percepción de los estudiantes sobre el desempeño docente pero con enfoques principalmente cuantitativos ${ }^{14}$.

Por este motivo, el objetivo de este estudio es explorar las percepciones de los estudiantes sobre sus vivencias durante las prácticas clínicas, ya que es un aspecto fundamental para tomar decisiones que permitan mejorar la calidad de la enseñanza.

\section{Método}

Es un estudio de abordaje metodológico descriptivo de tipo cualitativo y con un encuadre sociocrítico. Este paradigma desarrollado por Habermas ${ }^{15}$, adaptado al contexto educativo por diversos autores mantiene como unos de sus postulados esenciales transformar la reflexión crítica y la comunicación en instrumentos de cambio socioeducativo. Se estima de vital importancia la comunicación en un marco de reflexión crítica con la finalidad de optimizar el contexto global educativo. No sólo transforma la comunicación en instrumento de optimización de la realidad investigada, sino que fomenta la participación y se puede implicar al alumnado en un proceso de reflexión conjunta de los problemas de aprendizaje ${ }^{3}$. La metodología cualitativa utiliza el lenguaje como fuente esencial de la evidencia empírica, que centra su interés en los escenarios naturales y reales en los que los seres humanos interaccionan y se comunican.

Dicho estudio se desarrolla en la Escuela Universitaria de Enfermería de la Cruz Roja de Madrid. Se ha elegido para la selección de los participantes el muestreo por criterios o intencionado ${ }^{16}$. Son estudiantes de $4^{\circ}$ grado que se encuentran realizando sus prácticas clínicas en el curso 13-14. El motivo de dichos criterios para la selección es que $4^{\circ}$ grado es el curso académico del plan de estudios donde se encuentra 
la mayor carga de ECTS (European Credit Transfer and Accumulation System), ligados a las prácticas clínicas es el momento de mayor nivel de madurez intelectual predecible que presentan los estudiantes. En cuarto grado los estudiantes realizan las prácticas en unidades de servicios especiales (Enfermería en Cuidados Complejos), en unidades maternales e infantiles (Enfermería de la Mujer y Enfermería de la Infancia y la Adolescencia) y en centros de atención primaria (Enfermería Comunitaria). Los centros donde los estudiantes realizan las prácticas son el Hospital Universitario La Princesa, Hospital de la Cruz Roja San José y Santa Adela, Hospital Universitario Puerta de Hierro-Majadahonda, Hospital Universitario La Paz, Hospital Universitario Santa Cristina, Hospital Infantil Universitario del Niño Jesús, Centros de Atención Primaria y la Clínica Santa Elena.

Durante su estancia clínica, cada estudiante tiene asignado un tutor académico (profesor titular de la EUE Cruz Roja Madrid), que realiza su seguimiento a través de visitas-tutorías semanales en coordinación con el tutor profesional de cada centro sanitario. El tutor académico será el responsable del seguimiento y evaluación del portafolio de prácticas, mientras que el tutor profesional es el encargado de la evaluación de las prácticas dentro del centro.

De los 65 estudiantes que elaboraron sus portafolios fueron 45 los que quisieron participar en la presente investigación y firmaron el consentimiento informado. La detección de la recolección de datos vino determinada por la saturación teórica de los mismos. Se llega a la saturación cuando el fenómeno está suficientemente bien descrito y continuar no llevaría a obtener nuevos datos ${ }^{16}$.

La complejidad de las prácticas clínicas requiere el uso de instrumentos narrativos dado que estos facilitan una visión no reduccionista de dicha realidad, ya que favorecen la compresión y explicación de los fenómenos. La recopilación de la información se realizó a través de los portafolios de prácticas que realizaron los estudiantes.

El instrumento de recogida fue un portafolio semiestructurado, en el que se definió previamente las tareas o evidencias (Tabla 1), que reflejen tanto el proceso y los productos alcanzados por los estudiantes durante sus prácticas, como la reflexión realizada sobre su propio aprendizaje. El primer día del curso se les informó a los estudiantes los objetivos de las prácticas, las competencias a adquirir en cada estancia clínica y el procedimiento de seguimiento de las mismas, por parte de los tutores. Se les informó sobre los distintos aspectos de la metodología a utilizar.

Tabla 1. Diseño del portafolio de prácticas

\begin{tabular}{ll}
\hline Características & \multicolumn{1}{c}{ El portafolio de prácticas } \\
\hline \multirow{2}{*}{ Propósito } & $\begin{array}{l}\text { Centrado en el estudiante, en su actividad, reflexión y autoevaluaciones. } \\
\text { Presentación en formato electrónico a través de la plataforma Moodle. }\end{array}$ \\
\hline & $\begin{array}{l}\text { Fomentar la reflexión y pensamiento del estudiante. } \\
\text { Aprendizaje significativo. } \\
\text { La integración teórico-práctica. } \\
\text { Evaluación formativa y sumativa de las prácticas. }\end{array}$ \\
\hline Contenido & $\begin{array}{l}\text { Evidencias o muestras de aprendizaje individuales: reflexiones, síntesis, } \\
\text { incidentes críticos, propuestas de mejora y autoevaluaciones. }\end{array}$ \\
\hline Evaluación & $\begin{array}{l}\text { Puntuación dentro de la asignatura de prácticas: supone un } 30 \% \\
\text { de la nota final, que incluye su autoevaluación. }\end{array}$ \\
\hline
\end{tabular}

Las evidencias deben seguir las cuatro fases explicadas por Barberá ${ }^{17}$ : selección de evidencias relacionadas con la experiencia, identificación de las evidencias que sean susceptibles de demostrar que se está progresando en el aprendizaje, así como tener en cuenta los objetivos y competencias de la asignatura, reflexión sobre las evidencias además justificar por qué aporta esta evidencia y no otra y la relación con el aprendizaje intencionado, del mismo modo finalizar con la publicación de las evidencias acompañadas de la reflexión. Además, las tareas que se eligieron relacionándolas con los objetivos de aprendizaje a conseguir en los rotatorios de prácticas (Tabla 2). 


\begin{tabular}{ll}
\hline Estancia clínica & Evidencia del portafolio \\
\hline Centros de atención primaria & Revisión o elaboración de un programa de Educación para la Salud. \\
\hline Unidades especiales & $\begin{array}{l}\text { Incidente crítico. } \\
\text { Revisión bibliográfica sobre un tema relacionado con la unidad } \\
\text { (a elegir sin repetir en los dos rotatorios). }\end{array}$ \\
\hline Unidades materno infantiles & \\
\hline Especialidad a elegir por el estudiante & Informe final de prácticas. \\
\hline
\end{tabular}

Se realizó un análisis de contenido dividido en varias etapas: la reducción de datos (síntesis/procesamiento) a través de la elección del marco conceptual, luego se realizó la codificación con la catalogación-etiquetación de los fragmentos mediante palabras clave, lo que implica el siguiente nivel de reducción de datos, para posteriormente realizar la presentación de datos y conclusiones/verificación de los mismos. El proceso para alcanzar dicha forma de lectura vertebrada semánticamente fue el siguiente ${ }^{18}$ :

Preparación de los documentos primarios que son la base del análisis, es decir, los datos brutos.

Identificar las citas Quotations que son los segmentos significativos de los documentos primarios. Es la primera selección del material base del análisis.

Codificación de los documentos primarios: catalogación-etiquetación de los fragmentos mediante palabras clave, lo que correspondería a los temas emergentes de los discursos. Lo que implica el siguiente nivel de reducción de datos.

Escribir las anotaciones (memos) que habitualmente son comentarios asociados a una cita o un código, que es importante no olvidar y relacionar antes de hacer la interpretación.

Formar familias: agrupaciones de citas, documento o anotaciones vinculados por palabras clave, que tengan algún tipo de relación.

Finalmente, hacer representaciones gráficas o networks de las relaciones que se hayan establecido en la investigación.

Para el análisis se utilizó el programa Atlas-.Ti 6.0, que permitió, por un lado, reducir los datos recogidos y categorizarlos y, por otro lado, construir las conclusiones y reflexiones finales. Para la elaboración del estudio, se contó con el permiso del Comité Ético y de Investigación de la Universidad Autónoma de Madrid, así mismo se tomaron en cuenta los cuatro principios fundamentales de la bioética: autonomía, beneficencia, no maleficencia y justicia. Se enfatizó que su participación era libre y voluntaria, garantizándoles su derecho a abstenerse y retirar su participación en cualquier momento, así como en el manejo confidencial de la información obtenida. Se establecen la credibilidad, la transferibilidad y la auditabilidad como aspectos claves para asegurar la calidad de la investigación cualitativa. La triangulación de los resultados es un proceso que controla la subjetividad del investigador y valida los resultados que se obtengan dándole credibilidad. Además, la aplicación de los portafolios y recogida de la información estuvo siempre a cargo de los investigadores, puesto que ellos son los responsables del proceso de análisis e interpretación de los datos. Se considera que se ha cumplido la transferibilidad ya que se han recogido abundantes datos descriptivos, que dan información suficiente y representativa, que permiten comparar el contexto descrito con otros a los que se podría realizar transferencias. Conjuntamente, se ha realizado un registro y documentación completa de las decisiones e ideas que el investigador haya tenido en relación con el estudio. Esta estrategia permite que otro investigador examine los datos y pueda llegar a conclusiones iguales o similares a las del investigador original siempre y cuando tengan perspectivas similares.

\section{Resultados}

Las percepciones de los estudiantes sobre su experiencia en prácticas clínicas se agruparon en tres categorías: aprendizaje en prácticas, dificultades del entorno, crecimiento personal y profesional, dentro de las mismas se encuentran distintas unidades de significado (Tabla 3). 


\begin{tabular}{ll}
\hline Categorías & Unidades de Significado \\
\hline \multirow{2}{*}{ Aprendizaje en prácticas } & Importancia de las prácticas clínicas \\
& Fijación de conocimientos \\
& Aspectos negativos \\
\hline \multirow{2}{*}{ Dificultades del entorno } & Miedos, inseguridades \\
& $\begin{array}{l}\text { Papel del tutor de practicas } \\
\text { Rol de estudiante }\end{array}$ \\
\hline \multirow{2}{*}{ Crecimiento personal y profesional } & Construcción de su identidad profesional \\
& Visión holística del cuidado \\
\hline
\end{tabular}

\section{Aprendizaje en prácticas}

Es la temática que más unidades de significado recoge, ya que es a la cual los participantes han dedicado más reflexiones en sus portafolios. Este tema engloba todos aquellos aspectos referentes a la importancia de las prácticas clínicas, fijación de conocimientos teóricos-prácticos y aspectos negativos encontrados.

La mayoría indican que las prácticas clínicas son el eje fundamental e indispensable del grado en enfermería, ya que es donde conocen realmente la función del profesional enfermero:

Las prácticas han sido la parte más importante a lo largo de la carrera, es indudable que la teoría es muy necesaria, pero ésta no tendría ningún sentido si no se pudiera poner en práctica. Es durante este periodo donde te das cuenta de lo gratificante que puede resultar ayudar a las personas que lo necesitan y de la labor tan importante que desarrolla la enfermera en la sociedad, la cual no siempre es reconocida. P9:19.

De igual manera, consideran las prácticas clínicas como la oportunidad académica para aprender de verdad, afianzar sus conocimientos y ver la teoría del aula en casos reales, es decir, donde verdaderamente ponen en práctica todos sus conocimientos para encontrar el verdadero sentido a los mismos, las cuales sirven para su fijación a lo largo del tiempo:

Cierto es, que sin teoría no hay práctica, pero en mi opinión es en la práctica donde se afianzan todos los conceptos y habilidades teóricas estudiadas previamente. P12:18.

Pero también han identificado aspectos negativos, entre los que se encuentra el sistema de rotaciones por las unidades y las diferencias existentes en los cuidados según el turno. Consideran que no realizar las prácticas en el turno donde se realizan más cuidados influye negativamente en su aprendizaje:

El sistema de rotación por los diferentes centros y unidades, es práctico por una parte, porque conoces el funcionamiento de uno u otro hospital, clínica o centro de salud, y dentro de ellos, la gestión y administración de cuidados de las unidades que los componen; sin embargo, por la otra parte, es un hándicap hacia nuestro futuro profesional. Bajo mi punto de vista, como estamos tan poco tiempo en un hospital no nos llegan a conocer, es decir, nos tendrán menos o nada en cuenta a la hora de contratarnos. P3:10.

Además, recogen como aspectos negativos la duración de los rotatorios, debido a que consideran que son breves para poder aprovechar el máximo posible:

Siempre he pensado que los rotatorios deberían ser más largos, puesto que cuando comenzamos a controlar parte de lo que ocurre en el servicio en el que rotamos, nos cambian. P3:7.

\section{Dificultades del entorno}

El entorno clínico y la relación que se establezca entre este y el estudiante determina en muchas ocasiones el aprendizaje. Los estudiantes viven con mucha ilusión y expectativas el comienzo de sus prácticas clínicas, pero también les produce distintos sentimientos (miedo, inseguridad, dudas, estrés, intriga, timidez...) debido a su desconocimiento. En la mayoría de las ocasiones reflejan que estas percepciones se resolvieron al cabo de unas semanas, pero que precisaban de adaptación para familiarizarse con el entorno en la cual fue fundamental el apoyo del personal de las unidades:

Los cambios no son fáciles, llegar a un servicio o unidad diferente cada cierto intervalo de tiempo se acompañaba de un miedo más o menos explícito, al que iba relegando a un segundo plano a medida que adquiría seguridad en el nuevo entorno. La motivación, suponía el impulso que necesitaba para afrontar cada día de prácticas. Pero mentiría si no dijera que el recibimiento 
por parte del personal implicado hacía que ese miedo desapareciera o incluso dejara paso a otras emociones más constructivas antes de lo esperado en un principio o bien todo lo contrario. P17:50.

Sin embargo, esta integración en el equipo la consideran como un elemento motivador, proporcionándoles seguridad y sentimientos positivos.

Otra de las dificultades encontradas está relacionada con el rol del tutor profesional y el hecho de no tener un tutor fijo, ya que cada uno realiza las técnicas de diferente manera, por lo que tienen que adaptarse a las manías de cada uno de ellos, lo cual les generaba mucha inseguridad. Algunos describen haber aprendido una misma técnica de distintas maneras según el profesional que les tutorizaba. Esta situación les obstaculiza la aplicación de la teoría a la práctica:

También me ha resultado dificultoso el acordarme de cada una de las formas de poner en práctica la misma técnica dependiendo de los diferentes profesionales, además de resultarme una pérdida de tiempo en la mayoría de ocasiones porque los primeros rotatorios hasta llegue a anotar el nombre de la enfermera(o) y como realiza las cosas para evitar perder tiempo en el inicio de cada día, y sobre todo para evitar reprimendas porque he podido observar que en ocasiones está situación es un motivo de crítica hacía los estudiantes. P21:45.

En muchas ocasiones la dificultad va también relacionada con su rol de estudiante y algunos concretamente por sentirse jóvenes frente a distintas situaciones que han vivido en prácticas:

Como aspecto negativo únicamente puedo mencionar la limitación que supone el rol de estudiante, que en ocasiones no te permite actuar libremente, aunque lógico a la vez; y la presión de sentirse constantemente evaluado, ligada también al rol. P32:6.

\section{Crecimiento personal y profesional}

La mayoría de los estudiantes refieren que las prácticas han contribuido para su desarrollo académico y profesional, y también para crecer como persona y madurar, así como para crear su propio pensamiento e identidad:

Los rotatorios de prácticas clínicas han contribuido en mi forma de ser y de pensar. Evidentemente, las personas crecemos y maduramos con tiempo y experiencias. Para mí, es significativa la forma en que la enfermería ha cambiado mi forma de ser, los valores que tengo, mis modos de pensar y actuar. P36:15.

Exponen que desde el comienzo habían elegido esta profesión por vocación, sin embargo, eligieron esta titulación por otros motivos pero con el paso de los años han descubierto su interés:

Al principio pensaba que el centro de salud iba a ser muy monótono y aburrido y después de estas seis semanas salgo encan-

tada, me ha gustado mucho y me ha hecho replantearme muchas cosas sobre mis gustos para un futuro. P15:13.

No sólo describen la confirmación de ser enfermeros, sino que durante las prácticas han ido descubriendo sus gustos y posibles orientaciones profesionales. Manifiestan aquello que debe conseguir el estudiante para ser un buen profesional de enfermería, actuar y hacer como tal. Explican cómo ha ido modificándose su percepción de qué hace enfermería, por consiguiente ven en la profesión algo más que la simple realización de técnicas y así describen las funciones de enfermería:

La enfermera es la que observa, comunica, ayuda, enseña y atiende tanto al paciente como a su familia durante las $24 \mathrm{~h}$ del día; y es la que está a pie de cama brindando apoyo y empatía al paciente terminal. P12:15.

Además, expresan que las prácticas les han permitido concebir el cuidado como un proceso más allá de las meras técnicas y acciones dirigidas a la resolución física de la patología, así como tener en cuenta todos los aspectos bio-psico-sociales de las personas:

En el primer rotatorio me resultaba complicado estar pendiente de tantas cosas, recordar todo lo que envolvía al paciente...y posteriormente pude ver al paciente como un conjunto, pudiendo hacer una valoración mucho más completa y real. P30:41.

\section{Discusión}

Los estudiantes ponen de manifiesto que las prácticas clínicas son el eje fundamental del aprendizaje en enfermería, éstas son el contexto donde los conocimientos adquiridos cobran verdadero sentido. Concretamente, han reflexionado sobre lo que les aportan las prácticas clínicas, sus experiencias ante el primer contacto con el hospital y su integración con el equipo multidisciplinar. Estudios anteriores señalan 
que los estudiantes de enfermería refieren que no es posible adquirir un buen nivel de formación si no es a través de la experiencia clínica ${ }^{19,20}$. Por otro lado, manifiestan la importancia de una base teórica que permita identificar las acciones adecuadas, para llevar acabo la práctica utilizando el pensamiento crítico reflexivo. Consideran que si no existe esta buena base teórica no puede existir una buena práctica, ya que a partir de la teoría, además de utilizar su pensamiento crítico es cuando se realizan los cuidados de forma correcta. También, refieren que al comienzo de sus prácticas tuvieron mayor dificultad para integrar teoría y práctica, pero con el paso del tiempo han ido encontrando sentido todos sus conocimientos, así como realizar dicha integración de forma inconsciente. Queda patente que les permite tomar conciencia de aquellos conocimientos del saber, saber hacer y saber estar a lo largo de las prácticas, que son susceptibles de ser mejorados ${ }^{21}$.

Unas dificultades que encuentran es poder integrar los conocimientos en la práctica, en ocasiones incrementado por un desempeño inadecuado del rol del tutor profesional. En relación a este tema, algunos estudiantes indican que han tenido que adaptarse a los distintos profesionales, aunque en ocasiones la forma de realizar tal técnica o cuidado no fuera la correcta, lo cual influye directamente en su aprendizaje. Todo lo anterior concuerda con lo expuesto por distintos autores ${ }^{8,9}$, muestran que mantener un entorno de aprendizaje clínico positivo es de extrema importancia para asegurar el proceso de enseñanza-aprendizaje óptimo. Así como la importancia del papel del tutor asistencial a la hora de facilitar el aprendizaje de los estudiantes ${ }^{1,22,23}$.

Por otro lado, han reflexionado sobre lo que les aportan las prácticas clínicas, sus experiencias ante el primer contacto con el hospital y su integración con el equipo multidisciplinar. Reflejan las experiencias favorables que tienen durante sus estancias clínicas, que les permitan construir su identidad profesional, su desarrollo personal y tomar conciencia de la profesión. Estos resultados concuerdan con los aportados por Rivera ${ }^{14}$, donde se expresa que la inmersión del estudiante en la realidad hospitalaria le lleva a realizar una reflexión sobre sí mismo y sobre su futuro profesional.

\section{Conclusiones}

En conclusión, el estudio evidencia la importancia de las prácticas clínicas para los estudiantes dentro del plan de estudios, ya que es donde aplican lo que han aprendido en el aula y son conscientes de la realidad a la que se enfrentan. Para los estudiantes el rol del tutor de prácticas influencia directamente en su aprendizaje, tanto a nivel positivo como negativo. Las principales dificultades encontradas han estado relacionadas con el hecho de no tener un tutor fijo debido a las rotaciones y ser tutorizados por profesionales que no realizan los cuidados basándose en la evidencia científica o bien, que no tenían habilidades docentes necesarias.

\section{Responsabilidades éticas}

Protección de personas y animales. En esta investigación no se realizaron experimentos en seres humanos ni en animales.

Confidencialidad de los datos. Se aseguró y en el estudio no aparecen datos que permitan la identificación de los participantes.

Derecho a la privacidad y consentimiento informado. Se obtuvo consentimiento informado, se conservó el anonimato, y se respetó la integridad de los participantes.

Financiamiento. Ninguno.

Conflicto de intereses. Los autores declaran no tener conflicto de intereses.

Agradecimientos. Los autores quieren agradecer a todos/as los/as estudiantes de Grado en Enfermería que participaron en el estudio. 


\section{Referencias}

1. Fuentes-Pumarola C, Ballester-Ferrando D, Gelabert-Vilella S, Bosch-Farré C, Malagón-Aguilera MC, Rascón-Hernán C, et al. Nursing student and professor perceptions and assessments of the achievement of practicum competencies: A mixed method approach. Nurse Educ Today. 2016; 45: 199-205. https://doi.org/10.1016/j.nedt.2016.08.013

2. Zabalza-Beraza MA. La convergencia como oportunidad para mejorar la docencia universitaria. Rev. interuniv. form. profr. 2006; 20(3): 37-69. https://bit.ly/2YRRx5n

3. Siles-González J, Cibanal-Juan L, Domínguez-Santamaría JM, Pérez-Cañaveras RM, Vizcaya-Moreno MF, Gabaldón-Bravo EM, et al. Antropología educativa aplicada a las prácticas clínicas en enfermería: las diferencias culturales implicadas en el proceso de prácticas clínicas en enfermería. Una aportación desde la antropología de la complejidad y el pensamiento crítico. En: Martínez MA. (Coord.). Investigar en docencia universitaria. Redes de colaboración para el aprendizaje. Alcoy, España: Marfil. 2009. https://bit.ly/2NUbDe4

4. García-Rodríguez J. Las prácticas clínicas en los estudios de Grado en Enfermería. Enfermería Docente. 2013; 101: 2-3. https://bit.ly/32s2aOr

5. Sanjuán-Quiles Á, Martínez-Riera JR. Nuevo enfoque en el proceso de enseñanza-aprendizaje para la interrelación de conocimientos y formación de clínica/comunitaria. Inv. educ. enferm. 2008; XXVI(2 Sup): 150-9.

6. Spouse J. Bridging theory and practice in the supervisory relationship: a sociocultural perspective. J Adv Nurs. 2001; 33(4): 512-522. https://bit.ly/2YRVl6F

7. Albi-García J, Caamaño-Vaz MD, García-López F, Abad-Bernardo MA, Fernández-Vaca C, BenaventeSanguino MJ. Desarrollo de un instrumento de evaluación de las prácticas clínicas de los estudiantes de enfermería. Enferm. clín. 2003; 13(3): 146-53.https://doi.org/10.1016/S1130-8621(03)73797-8

8. Serrano-Gallardo P, Martínez-Marcos M, Espejo-Matorrales F, Arakawa T, Magnabosco GT, Pinto IC. Factors associated to clinical learning in nursing students in primary health care: an analytical crosssectional study. Rev Lat Am Enfermagem. 2016; 24: e2803.

https://doi.org/10.1590/1518-8345.0327.2803

9. Crespo-Knopfler S, González-Velázquez MS, Agama-Sarabia A. Percepción de los alumnos de enfermería sobre los factores que inciden en su aprendizaje clínico. Vertientes. 2011; 14(2): 88-93. https://bit.ly/2uQWwWY

10. Serrano-Gallardo P, Martínez-Marcos M. La tutorización de prácticas clínicas en pregrado de enfermería. Metas enferm. 2008; 11 (3): 28-32. https://bit.ly/2NoPAH1

11. Moreno-Tello MA, Prado-Moncivais EN, García-Avendaño DJ. Percepción de los estudiantes de enfermería sobre el ambiente de aprendizaje durante sus prácticas clínicas. rev. cuid. (Bucaramanga. 2010). 2013; 4(1): 444-9. https://bit.ly/2G8YE1V

12. Liljedahl M, Björck E, Kalén S, Ponzer S, Bolander-Laksov K. To belong or not to belong: nursing students' interactions with clinical learning environments-an observational study. BMC Med Educ. 2016; 16:197. https://doi.org/10.1186/s12909-016-0721-2

13. Tremayne P. Improving clinical placements through evaluation and feedback to staff. Nurs Times. 2007; 103(25): 32-3. https://bit.ly/2Llz7qn

14. Rivera-Alvarez LN. Saber y experiencia de el/la estudiante de enfermería en sus prácticas de cuidado [Tesis doctoral]. Barcelona: Universitat de Barcelona; 2013. https://bit.ly/2NUM2BH

15. Habermas J. Teoría y praxis: estudios de filosofía social. Madrid: Tecnos; 2002.

16. Hernández-Sampieri R, Fernández-Collado C, Baptista-Lucio P. Metodología de la Investigación. $5^{\text {ta }}$ ed. México: Mc Grall-Hill/Interamericana; 2006.

17. Barberá-Gregori E, de Martín-Rojo E. Portfolio electrónico: aprender a evaluar el aprendizaje. Barcelona: UOC; 2009. 
18. Solano-Ruiz MC. Vivencias de las personas que han padecido un infarto agudo de miocardio tras un año de evolución un estudio desde la perspectiva de la fenomenología social desarrollada en el Departamento de Salud número 20 de la Comunidad Valenciana (Tesis doctoral). España: Universidad Alicante; 2007.

19. Lorente-Gallego AM, García-Sánchez C, López-Arroyo MJ, Echevarria-Pérez P, Morales-Moreno I. Satisfacción de los alumnos de enfermería de la Universidad Católica San Antonio (UCAM) sobre su formación práctica. Seminarios en salas de demostraciones y prácticas clínicas. Enfermería glob. 2009; 17: 1-11. https://bit.ly/2XSZYMq

20. Uriarte $\mathrm{S}$, Ponce $\mathrm{G}$, Bernal M. Vivencias cotidianas en espacios clínicos del estudiante de enfermería. Enferm. univ. 2016; 13(3): 171-7. http://dx.doi.org/10.1016/j.reu.2016.07.002

21. Vizcaya-Moreno MF. Valoración del entorno de aprendizaje clínico hospitalario desde la perspectiva de los estudiantes de enfermería. (Tesis doctoral). España: Universidad de Alicante; 2005.

22. Castillo-Parra S, Vessoni-Guioti RD. La relación tutor-estudiante en las prácticas clínicas y su influencia en el proceso formativo del estudiante de Enfermería. Educare21. 2007; 38. http://repositorio.uchile.cl/handle/2250/128534

23. Márquez-Martínez G, Morán-Peña SE, Pérez-Zumano, SE. Vivencias de los pasantes de enfermería en la resolución de problemas clínicos. Enferm. univ. 2016; 13(1): 31-9.

https://doi.org/10.1016/j.reu.2016.01.003 